\title{
A Study of Novel Febrile Neutropenia Risk Factors Related to Bone Marrow or Immune Suppression, Barrier Function, and Bacterial Flora
}

\author{
Leila Family, PhDa; Yanli Li, PhD ${ }^{\text {; }}$ Lie Hong Chen, DPH; John H. Page, MD, ScDd; Zandra K. Klippel, MDe; \\ and Chun Chao, $\mathrm{PhD}^{\mathrm{c}}$
}

\begin{abstract}
Background: Previously identified patient-level risk factors for chemotherapy-induced febrile neutropenia (FN) indicate several potential underlying pathogenic mechanisms, including bone marrow suppression, impaired neutrophil function, or disturbances of barrier function. This study evaluated whether additional clinical characteristics related to these pathogenic mechanisms were risk factors for FN. Patients and Methods: The study population included patients diagnosed with non-Hodgkin's lymphoma or breast, lung, colorectal, ovarian, or gastric cancer between 2000 and 2009 at Kaiser Permanente Southern California and treated with myelosuppressive chemotherapy. Those who received prophylactic granulocyte colony-stimulating factor or antibiotics were excluded. Potential risk factors of interest included surgery, radiation therapy, selected dermatologic/mucosal conditions, and use of antibiotics and corticosteroids. All data were collected using electronic medical records. Multivariable Cox models were used to evaluate associations between these factors and risk of FN in the first chemotherapy cycle, and adjusted using propensity score-based functions. Results: A total of 15,971 patients were included. Of these, $4.3 \%$ developed FN in the first chemotherapy cycle. Use of corticosteroids was significantly associated with increased risk of FN (adjusted hazard ratio [aHR], 1.53; 95\% Cl, 1.17-1.98). Selected dermatologic/mucosal conditions and intravenous antibiotic use were marginally associated with increased risk of $\mathrm{FN}(\mathrm{aHR}, 1.40 ; 95 \% \mathrm{Cl}, 0.98-1.93$, and 1.35; 95\% Cl, 0.97-1.87, respectively). Surgery, radiation therapy, and oral antibiotic use were not statistically significantly associated with FN. Conclusions: Dermatologic or mucosal conditions that might affect barrier integrity and use of corticosteroids and intravenous antibiotics prior to chemotherapy may increase risk of FN and should be considered in prophylaxis use and FN prediction modeling.
\end{abstract}

J Natl Compr Canc Netw 2018;16(10):1201-1208 doi: 10.6004/jncen.2018.7051

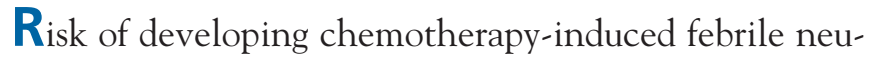
tropenia (FN) depends on patient-, treatment-, and disease-related characteristics. ${ }^{1}$ In our prior investigation, several chronic comorbidities were associated with significantly increased FN risk, including chronic obstructive pulmonary disease (COPD), congestive heart failure (CHF), autoimmune diseases, osteoarthritis, peptic ulcer disease, thyroid disorder, renal disease, liver

\footnotetext{
a Office of Health Assessment and Epidemiology, Los Angeles County Department of Public Health, Los Angeles; ${ }^{b}$ Center for Observational Research, Amgen Inc., South San Francisco; 'Department of Research and Evaluation, Kaiser Permanente Southern California, Pasadena; and ${ }^{\mathrm{d} C e n t e r}$ for Observational Research, and eHematology/Oncology, Amgen Inc., Thousand Oaks, California.

Submitted October 12, 2017; accepted for publication June 11, 2018. Drs. Li, Page, and Klippel have disclosed that they are employees and stock holders of Amgen, Inc. Drs. Family, Chen, and Chao have disclosed that they received research funding from Amgen, Inc. to perform this study. This work is supported by Amgen, Inc. (\#135884). All procedures
}

disease, and HIV infection. ${ }^{2,3}$ We hypothesized that the increased FN risk associated with these comorbidities may be mediated at least in part through the following biological mechanisms: bone marrow suppression, impaired neutrophil and other immune cell function, disturbances of barrier function/increased availability of pathogenic microbes, and impaired bacterial flora. ${ }^{2}$ For example, renal disease and thyroid disorder have been

performed in studies involving human participants were in accordance with the ethical standards of KPSC's Institutional Review Board and with the 1964 Helsinki Declaration and its later amendments or comparable ethical standards. For this type of study, formal consent is not required. Author contributions: Study concept and design: Chao, Li, Page, Family. Data acquisition and statistical analysis: Family, Chao, Chen. Guidance to data collection and analysis: Page, Li, Klippel. Manuscript preparation: Family, Li, Chen. Critical review and revision: All authors.

Correspondence: Chun Chao, PhD, Department of Research and Evaluation, Kaiser Permanente Southern California, 100 South Los Robles Avenue, 2nd Floor, Pasadena, CA 91101. Email: Chun.R.Chao@kp.org 
Family et al

associated with impaired neutrophil function. ${ }^{4-6}$ Peptic ulcer disease and COPD may be linked to FN via disturbances of barrier function, impaired bacterial flora, and, in the case of COPD, use of inhaled steroids. $^{7-9}$

Based on these previously hypothesized underlying pathogenic mechanisms for FN, we further hypothesize that other clinical conditions that potentially lead to one or more of these pathogenic states may also increase risk of FN during chemotherapy. Specifically, we hypothesize that surgery, radiation therapy (RT), use of corticosteroids and antibiotics, and certain dermatologic/mucosal conditions might increase risk of chemotherapy-induced FN (hypothesized mechanisms and associated conditions are detailed in supplemental eTable 1, available with this article at JNCCN.org). Prior or concurrent RT may predispose patients with cancer to infections via bone marrow suppression and damage of mucosal barrier. ${ }^{10-12}$ Recent surgery and select dermatologic and mucosal conditions could indicate a breakdown in skin/mucosal integrity and increased exposure to pathogenic microbes. ${ }^{13,14}$ Antibiotic use prior to chemotherapy could alter bacterial flora. ${ }^{15,16}$ Corticosteroids are known to impair immune cell function. ${ }^{17}$

NCCN recommendations for prophylactic management of FN depends on the expected FN risk level the patient will experience, and accurate prediction of a given patient's FN risk depends on knowledge of key FN risk factors. The performance of currently available FN prediction models in the literature indicated room for improvement in clinical FN risk prediction. ${ }^{18,19}$ Thus, the objective of this study was to assess the effects of the aforementioned clinical conditions on FN risk during chemotherapy to shed light on novel FN risk factors that may be considered in clinical FN risk prediction.

\section{Patients and Methods}

\section{Study Setting and Population}

Kaiser Permanente Southern California (KPSC) is an integrated healthcare delivery system that provides comprehensive health services for 4 million enrollees who are representative of the racial/ethnic diversity in Southern California. ${ }^{20}$ KPSC maintains electronic medical records (EMRs) for virtually all aspects of care delivered, all linkable through a unique member identifier. The study cohort was composed of patients who were (1) diagnosed with non-Hodgkin's lymphoma (NHL) or breast, lung, colorectal, ovarian, or gastric cancer at KPSC between January 1, 2000, and December 31, 2009; (2) aged $\geq 18$ years at diagnosis; (3) initiated myelosuppressive chemotherapy (defined per reference ${ }^{21}$ ) within 1 year of diagnosis; and (4) KPSC members for at least 12 months prior to diagnosis. These cancer types were chosen because they were relatively common and would be managed with myelosuppressive chemotherapy agents. Patients who met the following criteria were excluded: (1) had unknown cancer stage, chemotherapy agents, or cycle length; (2) received prophylactic granulocyte colony-stimulating factor (G-CSF) within 4 days of chemotherapy initiation and/or antibiotics prophylaxis dispensed between 3 days before and 10 days after first chemotherapy administration in the absence of fever/infection; (3) received dose-dense chemotherapy regimens or weekly regimens; (4) had bone marrow/stem cell transplantation; or (5) were patients of Orange County Medical Center if their cancer was diagnosed before 2007, for whom data on absolute neutrophil count (ANC) could not be readily accessed via EMR.

\section{Data Collection}

Exposures of interest were surgery prior to chemotherapy, RT before or concurrent with chemotherapy, corticosteroid and antibiotic use prior to chemotherapy, and selected dermatologic and mucosal conditions. Prior surgery was defined as cancerrelated excisional treatment procedures received within 6 months prior to chemotherapy initiation. Prior and concurrent RT were defined as RT administered within 2 months prior to chemotherapy and within 5 days after chemotherapy initiation, respectively. A 2-month window was chosen for prior RT because previous studies showed that WBC counts did not return to normal until 6 to 8 weeks after radiation exposure. ${ }^{12,22}$ Corticosteroid use was identified within 3 months prior to chemotherapy initiation. Because most corticosteroid use by the study population was oral prescriptions $(88 \%)$, only oral corticosteroid use was evaluated in this study. Antibiotic use was defined as at least 5 days of continuous outpatient antibiotic prescriptions, with the last day of use within 3 months prior to chemotherapy initiation. A 3-month window was chosen because previous studies showed that bacterial flora became nor- 
Risk Factors for Febrile Neutropenia

malized 3 months after withdrawal of antibiotics. ${ }^{23}$ Only outpatient oral antibiotic use was considered in the main analysis. A subanalysis evaluating both oral and any duration of intravenous antibiotic use from both outpatient and inpatient settings was limited to 2008 to 2009, when data on inpatient antibiotic use became assessable electronically. Selected dermatologic and mucosal conditions likely to damage the integrity of the barrier function were assessed using ICD-9 codes within 1 month prior to chemotherapy. The specific data source, ICD-9 diagnosis, and CPT codes to identify exposures of interest are shown in supplemental eTable 2 .

The outcome of interest was the occurrence of FN within the first cycle of chemotherapy. FN was defined using one of the following methods: (1) neutropenia ICD-9 code 288.0 and fever ICD-9 code 780.6 within 7 days; (2) ANC $<1,000 / \mathrm{mcL}$ and fever ICD-9 code 780.6 within 7 days; (3) hospitalization with neutropenia, ICD-9 code 288.0, as the primary diagnosis; (4) neutropenia ICD-9 code 288.0 or ANC $<1,000 / \mathrm{mcL}$ and hospitalization with ICD-9 code of bacterial/fungal infection within 7 days; or (5) neutropenia ICD-9 code 288.0 or ANC $<1,000 /$ $\mathrm{mcL}$ and intravenous antibiotic use within 7 days. When not specified, both inpatient and outpatient corresponding ICD-9 codes were used to capture FN outcomes. FN outcomes were assessed only in the first chemotherapy cycle to allow the most unbiased assessment of the effect of new risk factors on FN. Subjects were followed from the first chemotherapy administration to the FN event, the beginning of the second chemotherapy cycle, termination of KSPC membership, death, or 28 days after the first chemotherapy administration, whichever came first.

Patient characteristics included age at diagnosis, sex, race/ethnicity, census block income and education level, Medicare or Medicaid enrollment, and length of KPSC membership. Cancer and treatment characteristics included stage at diagnosis and chemotherapy regimens, which were categorized based on its risk of inducing $\mathrm{FN}$ into highly myelosuppressive ( $>20 \%$ risk of $\mathrm{FN})$, moderately myelosuppressive $(10 \%-20 \%$ risk $)$, or unclassified $(<10 \%$ risk and all other regimens not in these lists). ${ }^{24}$ In addition, the number of myelosuppressive agents used with and without dose reduction (ie, received $<90 \%$ of standard dose based on body surface area) was also considered. Laboratory measures of interest included
ANC at the time of diagnosis. Comorbidities previously shown to increase the risk of $\mathrm{FN}$ were captured, including COPD, CHF, HIV infection, rheumatoid diseases, other autoimmune disorder, thyroid disorder, peptic ulcer disease, diabetes, renal failure, and liver disease. Recent infection history, defined as any bacterial or fungal infection within 3 months prior to chemotherapy initiation, was also captured. Both comorbidities and infection history were ascertained using ICD-9 codes.

\section{Statistical Analysis}

Distributions of patient characteristics and occurrence of FN were described overall and by cancer type. Bivariable and multivariable Cox models were used to evaluate the association between each of the potential risk factors of interest and occurrence of FN in the first chemotherapy cycle. For the multivariable analysis, we used propensity score functions to adjust for potential confounders. The following variables were included in the propensity score function for each potential risk factor of interest: age, sex, race/ethnicity, length of KPSC membership, public insurance status, census level socioeconomic indicators, total number of comorbidities, recent infection, previous cancer, and cancer stage at diagnosis (Table 1 footnote). An additional 12 subjects were excluded from the multivariable analysis due to missing data on census level socioeconomic status. The distribution of these confounders by exposure status within propensity score quintiles was examined to determine if balance was achieved. Additional confounders that are specific to each potential risk factor of interest were also selected based on prior knowledge and adjusted in the propensity score function (see Table 1 footnote). The propensity score was included as a covariate (smoothed with natural cubic splines) in the Cox model to assess the effect of potential risk factors of interest on FN. The analyses were stratified by cancer type using the "strata" comment in SAS Phreg to account for potential confounding by cancer type.

Alternative models that additionally adjusted for potential confounders that may be on the causal pathway, such as baseline ANC, baseline anemia (defined as prior diagnosis of anemia or baseline hemoglobin $<10 \mathrm{~g} / \mathrm{dL}$ ), chemotherapy regimen FN risk group, number of myelosuppressive agents with dose reduced and not reduced, were also evaluated in sen- 
Family et al

\begin{tabular}{|c|c|c|c|c|c|c|c|}
\hline \multirow[b]{2}{*}{ Potential Risk Factors for FN } & \multirow{2}{*}{$\begin{array}{l}\text { Number With } \\
\text { FN/Number of } \\
\text { Patients (\%) }\end{array}$} & \multicolumn{2}{|c|}{ Bivariate Cox Model } & \multicolumn{2}{|c|}{ PS-Adjusted Modela ${ }^{2}$} & \multicolumn{2}{|c|}{ Sensitivity Analysis ${ }^{\mathrm{b}}$} \\
\hline & & HR $(95 \%$ Cl) & $P$ Value & HR $(95 \% \mathrm{Cl})$ & $P$ Value & HR $(95 \% \mathrm{Cl})$ & $P$ Value \\
\hline \multicolumn{8}{|l|}{ Prior surgery } \\
\hline No & $351 / 6,355(5.5 \%)$ & Ref & & Ref & & Ref & \\
\hline Yes & $342 / 9,616(3.6 \%)$ & $0.86(0.70-1.06)$ & .15 & $0.89(0.72-1.11)$ & .30 & $0.99(0.80-1.23)$ & .95 \\
\hline \multicolumn{8}{|l|}{ Prior RT } \\
\hline No & $655 / 14,910(4.4 \%)$ & Ref & & Ref & & Ref & \\
\hline Yes & $38 / 1,061(3.6 \%)$ & $0.92(0.64-1.27)$ & .62 & $0.91(0.64-1.27)$ & .61 & $0.95(0.66-1.32)$ & .75 \\
\hline \multicolumn{8}{|l|}{ Concurrent RT } \\
\hline No & $678 / 15,549(4.4 \%)$ & Ref & & Ref & & Ref & \\
\hline Yes & $15 / 422(3.6 \%)$ & $0.99(0.56-1.61)$ & .96 & $1.32(0.69-2.37)$ & .37 & $1.27(0.67-2.26)$ & .43 \\
\hline \multicolumn{8}{|l|}{ Oral corticosteroid use } \\
\hline No & $624 / 15,083(4.1 \%)$ & Ref & & Ref & & Ref & \\
\hline Yes & $69 / 888(7.8 \%)$ & $1.71(1.32-2.20)$ & $<.01$ & $1.53(1.17-1.98)$ & $<.01$ & $1.49(1.13-1.93)$ & $<.01$ \\
\hline \multicolumn{8}{|l|}{ Oral antibiotic use } \\
\hline No & $390 / 8,946(4.4 \%)$ & Ref & & Ref & & Ref & \\
\hline Yes & $303 / 7,025(4.3 \%)$ & $1.06(0.92-1.24)$ & .42 & $0.99(0.85-1.16)$ & .94 & $1.00(0.86-1.17)$ & .98 \\
\hline \multicolumn{8}{|c|}{ Selected dermatologic and mucosal conditions } \\
\hline No & $656 / 15,494(4.2 \%)$ & Ref & & Ref & & Ref & \\
\hline Yes & $37 / 477(7.8 \%)$ & $1.58(1.11-2.17)$ & .01 & $1.40(0.98-1.93)$ & .05 & $1.26(0.88-1.74)$ & .19 \\
\hline
\end{tabular}

Abbreviations: CHF, congestive heart failure; COPD, chronic obstructive pulmonary disease; FN, febrile neutropenia; HR, hazard ratio; KPSC, Kaiser Permanente Southern California; PS, propensity score; RT, radiation therapy.

aThe following variables were included in the propensity score function for each potential risk factor of interest: age (modeled as natural cubic splines), sex, race/ethnicity, length of KPSC membership (in linear form), public insurance status (Medicare enrollees and Medicaid enrollees), socioeconomic indicators (including census track median annual household income: $\$ 0-\$ 25,000, \$ 25,001-\$ 40,000, \$ 40,001-\$ 60,000, \$ 60,001-\$ 80,000$, and $>\$ 80,000$; and proportion of adults with a high school diploma or a higher degree in the census track: $\leq 50 \%, 51 \%-75 \%,>75 \%)$, total number of comorbidities, previous cancer, stage at diagnosis, and recent infection. Additional confounders that are specific to each potential risk factor of interest were also adjusted in the propensity score function, including surgery (as a potential confounder) for prior RT; RT for prior surgery; diabetes, COPD, renal disease, autoimmune disorder (including thyroid disease and rheumatoid arthritis), HIV infection, prior surgery and prior RT, obesity, diabetes, CHF, osteoarthritis for antibiotic use; diabetes, HIV infection, autoimmune disorder, prior RT and prior surgery for dermatologic/mucosal conditions; baseline absolute neutrophil count, chemotherapy regimen FN risk category (high, medium, low/undefined), number of myelosuppressive agents dose-reduced and not dose-reduced, prior RT, and prior surgery for concurrent RT; and CHF, COPD, rheumatoid disease, osteoarthritis, other autoimmune disease, peptic nuclear disease, thyroid disorder for oral prior RT, and prior
corticosteroid use.

${ }^{\mathrm{b}}$ Additionally adjusted for covariates that may be on the causal pathway.

sitivity analyses. In another sensitivity analysis, antibiotic use (both intravenous and oral) within 7 days of surgery was also adjusted for in the model evaluating the association between surgery and FN, limiting to 2008 through 2009.

We also evaluated the effect of corticosteroid by duration of use (no use, <15 days, 15-29 days, 30-44 days, and 45-90 days) and recentness of use (no use, $<15$ days, 15-29 days, 30-44, and 45-90 days prior to chemotherapy). Alternative recentness of use was also assessed for oral antibiotic use $(\leq 30$ days and $<15$ days prior to chemotherapy), as well as for prior surgery ( $\leq 30$ days prior to chemotherapy). All analyses were performed with SAS 9.3 (SAS Institute Inc.).

\section{Results}

A total of 62,714 adult patients were diagnosed with 1 of 6 cancer types of interest between 2000 and 2009 at KPSC. Of these, 15,971 were included in the analysis after applying the eligibility criteria. Figure 1 shows population dispositions after applying each inclusion and exclusion criterion. Table 2 presents the distribution of demographic and clinical characteristics of the study population. Prior surgery $(60.2 \%)$ and oral antibiotic use $(44.0 \%)$ were common in the study population. RT was received by $6.6 \%$ and $2.6 \%$ of the study population in the period prior to and concurrent with chemotherapy, respectively. Oral corticosteroid use was found in $5.6 \%$ of patients prior to chemotherapy. Selected dermatologic and mucosal conditions were found in $3.0 \%$ of patients.

Overall, $4.3 \%$ of the study patients developed $\mathrm{FN}$ in the first chemotherapy cycle. Table 1 presents the incidence proportions of FN in the first chemotherapy cycle for patients with and without each of the potential risk factors. The propensity score-adjusted hazard ratio (aHR) estimates for FN risk associated with each of the potential risk factors are also shown in Table 1 . Selected dermatologic/mucosal conditions were marginally associated with increased risk of FN with statistical significance (aHR, 1.40; 95\% CI, 0.98-1.93). Corticosteroid use overall was associated with a statistically significant increase in FN risk (aHR, 1.53; 95\% CI, 1.17-1.98). A positive relationship was observed for the dura- 
Risk Factors for Febrile Neutropenia

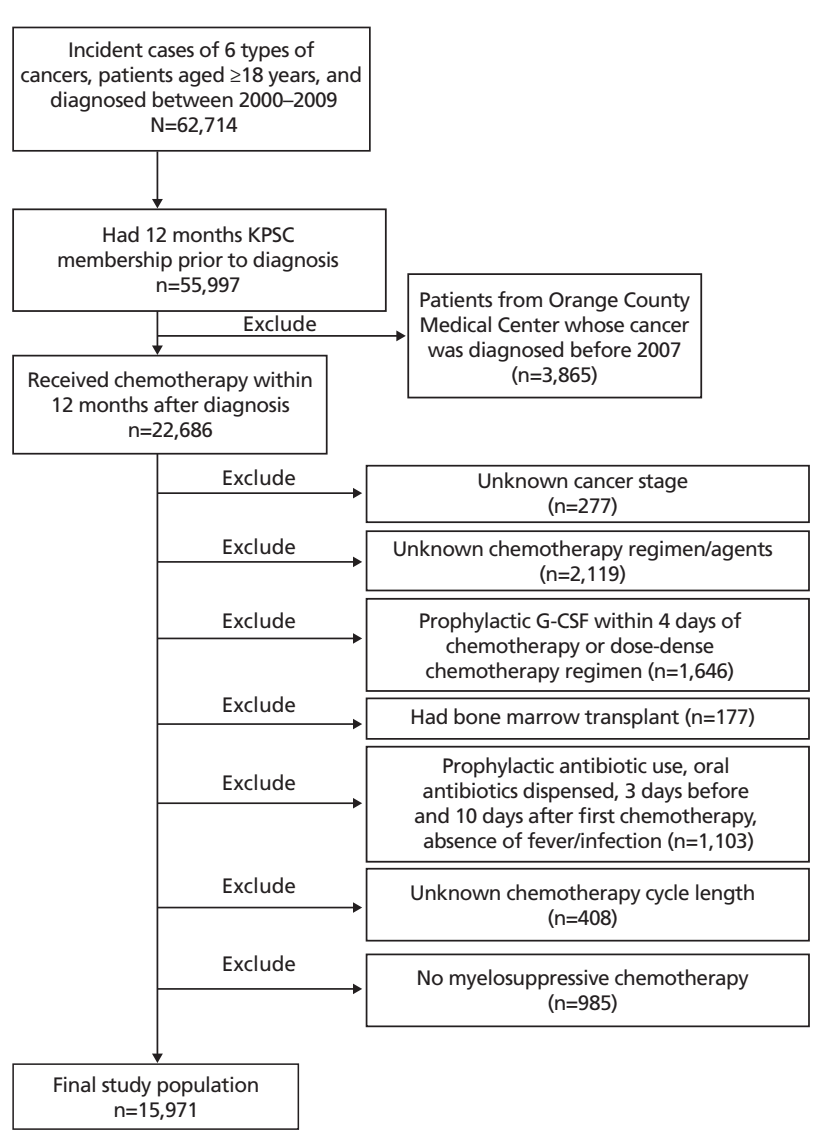

Figure 1. Study population flow chart.

Abbreviations: G-CSF, granulocyte colony-stimulating factor; KPSC, Kaiser Permanente Southern California.

tion of corticosteroid use and FN risk, with the aHR increasing from 1.78 (95\% CI, 1.50-2.11) for use of $<15$ days to 2.86 (95\% CI, 1.74-4.47) for use of 45 to 90 days (Table 3). However, no clear pattern was observed with the recentness of corticosteroid use. For antibiotic use, no association was observed for oral antibiotic use in the main analysis nor in the sensitivity analysis restricting the use to $\leq 30$ days or $<15$ days prior to chemotherapy (data not shown). However, in the subanalysis including data on inpatient antibiotic use, intravenous antibiotic use (aHR, 1.35; 95\% CI, 0.97-1.87) but not oral antibiotic use (aHR, 1.07; 95\% CI, 0.77-1.48) was marginally associated with increased risk of FN relative to no antibiotic use (Table 4).

No other potential risk factors examined were associated with risk of FN (Table 1). For prior surgery, no clear association was observed when exposure was restricted to $\leq 30$ days prior to chemotherapy (aHR, 1.03; 95\% CI, 0.82-1.28; data not shown). Similar results were obtained from all the sensitivity analyses additionally adjusted for potential confounders that could be on the causal pathways (Table 1).

Balanced distribution was achieved for most but not all measured confounders for each exposure of interest on examination within the propensity score quintiles (supplemental eTables 3-8).

\section{Discussion}

We found that corticosteroid use, selected dermatologic/mucosal conditions, and intravenous antibiotic use were associated with increased FN risk. Our findings on corticosteroid use are consistent with those reported by Lyman et $\mathrm{al},{ }^{18}$ who found that use of immunosuppressive drugs was a risk factor for $\mathrm{FN}$. We further found that a duration response relationship might exist for corticosteroid use, and even a short period of use (ie, $<15$ days) was significantly associated with increased FN risk. These findings have particular relevance for treatment regimens in which corticosteroids are often used prior to the start of chemotherapy. Our findings on the selected dermatologic/mucosal conditions and intravenous antibiotic use are novel, and to our knowledge, no prior study has examined the effect of these factors on FN risk. The results of this study suggest that corticosteroid use, intravenous antibiotics, and selected dermatologic and mucosal conditions should be taken into consideration when evaluating the need for prophylactic G-CSF or dose reduction, and during patient monitoring.

There is biological plausibility to support the associations observed in our study. Corticosteroid use suppresses immune cell function, and hence increases host susceptibility to pathogenic microbes. Several studies have shown that corticosteroids reduce neutrophils' adherence to endothelium, inhibiting migration to inflammatory sites. ${ }^{25}$ In hematologic malignancies such as NHL, corticosteroids are often used in high doses, increasing the risk of infection with a broad spectrum of pathogens. ${ }^{11}$ In addition to NHL, corticosteroids were also commonly used prior to chemotherapy among patients with lung cancer (14\%) in our study. Therefore, our findings may have important implications for FN prevention in these cancers. Although our examination was limited to use prior to chemotherapy, our results suggest that 
Family et al

\begin{tabular}{|c|c|c|c|c|c|c|c|}
\hline Characteristics & NHL & Breast Cancer & Lung Cancer & Colorectal Cancer & Ovarian Cancer & Gastric Cancer & Overall \\
\hline Number & 1,617 & 6,323 & 3,584 & 3,062 & 924 & 461 & 15,971 \\
\hline \multicolumn{8}{|l|}{ Demographics } \\
\hline Mean age at diagnosis (SD), y & $63.6(13.9)$ & $55.4(10.6)$ & $65.6(9.5)$ & $61.7(11.6)$ & $59.6(13.1)$ & $59.9(11.7)$ & $60.1(11.9)$ \\
\hline Female sex & $743(46.0 \%)$ & $6,294(99.5 \%)$ & $1,597(44.6 \%)$ & $1,408(46.0 \%)$ & $924(100 \%)$ & $151(32.8 \%)$ & $11,117(69.6 \%)$ \\
\hline \multicolumn{8}{|l|}{ Race/Ethnicity } \\
\hline Non-Hispanic white & $1,051(65.0 \%)$ & $3,615(57.2 \%)$ & $2,482(69.3 \%)$ & $1,888(61.7 \%)$ & $611(66.1 \%)$ & $215(46.6 \%)$ & $9,862(61.8 \%)$ \\
\hline Non-Hispanic black & $132(8.2 \%)$ & $943(14.9 \%)$ & $530(14.8 \%)$ & $435(14.2 \%)$ & $72(7.8 \%)$ & $62(13.5 \%)$ & $2,174(13.6 \%)$ \\
\hline Asian & $131(8.1 \%)$ & $691(10.9 \%)$ & $285(8.0 \%)$ & $276(9.0 \%)$ & $95(10.3 \%)$ & $59(12.8 \%)$ & $1,537(9.6 \%)$ \\
\hline Hispanic & $293(18.1 \%)$ & $1,033(16.3 \%)$ & $260(7.3 \%)$ & $438(14.3 \%)$ & $143(15.5 \%)$ & $121(26.3 \%)$ & $2,288(14.3 \%)$ \\
\hline Other/Unknown & $10(0.6 \%)$ & $41(0.7 \%)$ & $27(0.8 \%)$ & $25(0.8 \%)$ & $3(0.3 \%)$ & $4(0.9 \%)$ & $110(0.7 \%)$ \\
\hline Mean length of membership (SD), y & $18.0(12.7)$ & $16.2(11.4)$ & $17.9(12.1)$ & $17.5(12.5)$ & $17.0(12.1)$ & $16.2(12.2)$ & $17.1(12.0)$ \\
\hline Medicare enrollees & $750(46.4 \%)$ & $1,167(18.5 \%)$ & $1,850(51.6 \%)$ & $1,146(37.4 \%)$ & $310(33.6 \%)$ & $166(36.0 \%)$ & $5,389(33.7 \%)$ \\
\hline Medicaid enrollees & $11(0.7 \%)$ & $73(1.2 \%)$ & $25(0.7 \%)$ & $34(1.1 \%)$ & $8(0.9 \%)$ & $4(0.9 \%)$ & $155(1.0 \%)$ \\
\hline \multicolumn{8}{|l|}{ Cancer characteristics } \\
\hline \multicolumn{8}{|l|}{ Stage at diagnosis } \\
\hline Localized & $424(26.2 \%)$ & $2,980(47.1 \%)$ & $186(5.2 \%)$ & $429(14.0 \%)$ & $124(13.4 \%)$ & $39(8.5 \%)$ & $4,182(26.2 \%)$ \\
\hline Regional & $324(20.0 \%)$ & $3,089(48.9 \%)$ & $1,031(28.8 \%)$ & $1,826(59.6 \%)$ & $205(22.2 \%)$ & $232(50.3 \%)$ & $6,707(42.0 \%)$ \\
\hline Distant & $869(53.7 \%)$ & $254(4.0 \%)$ & $2,367(66.0 \%)$ & $807(26.4 \%)$ & $595(64.4 \%)$ & $190(41.2 \%)$ & $5,082(31.8 \%)$ \\
\hline \multicolumn{8}{|l|}{ Chemotherapy characteristics } \\
\hline \multicolumn{8}{|l|}{ Chemotherapy regimen FN risk group } \\
\hline High & $14(0.9 \%)$ & $257(4.1 \%)$ & $0(0.0 \%)$ & $1(0.0 \%)$ & $3(0.3 \%)$ & $17(3.7 \%)$ & $292(1.8 \%)$ \\
\hline Medium & $1,145(70.8 \%)$ & 4,933 (78.0\%) & $3,256(90.9 \%)$ & $1,374(44.9 \%)$ & $877(94.9 \%)$ & $136(29.5 \%)$ & $11,721(73.4 \%)$ \\
\hline Unclassified & $458(28.3 \%)$ & $1,133(17.9 \%)$ & $328(9.2 \%)$ & $1,687(55.1 \%)$ & $44(4.8 \%)$ & $308(66.8 \%)$ & $3,958(24.8 \%)$ \\
\hline Dose reduction, yes (any agent) & $386(23.9 \%)$ & $1,058(16.7 \%)$ & $1,002(28.0 \%)$ & $659(21.5 \%)$ & $130(14.1 \%)$ & $107(23.2 \%)$ & $3,342(20.9 \%)$ \\
\hline Myelosuppressive agents, mean (SD) & $1.8(0.5)$ & $1.9(0.7)$ & $1.8(0.4)$ & $1.0(0.1)$ & $1.9(0.3)$ & $1.4(0.6)$ & $1.7(0.7)$ \\
\hline Number dose reduced, mean (SD) & $0.3(0.6)$ & $0.2(0.5)$ & $0.3(0.5)$ & $0.2(0.4)$ & $0.1(0.4)$ & $0.3(0.7)$ & $0.2(0.5)$ \\
\hline Number not dose reduced, mean (SD) & $1.5(0.8)$ & $1.7(0.9)$ & $1.5(0.7)$ & $0.8(0.4)$ & $1.8(0.5)$ & $1.1(0.7)$ & $1.4(0.8)$ \\
\hline \multicolumn{8}{|l|}{ Laboratory measurements at diagnosis } \\
\hline Mean neutrophil count (SD), $1,000 / \mathrm{mcL}$ & $5.5(3.7)$ & $4.6(2.3)$ & $7.0(3.9)$ & $5.0(2.6)$ & $5.5(3.4)$ & $5.5(3.0)$ & $5.4(3.2)$ \\
\hline Recent infection & $373(12.18 \%)$ & $171(18.51 \%)$ & $64(13.88 \%)$ & $1,970(12.33 \%)$ & $171(18.51 \%)$ & $64(13.88 \%)$ & $1,970(12.33 \%)$ \\
\hline \multicolumn{8}{|l|}{ Exposure of interest } \\
\hline Prior surgery & $215(13.3 \%)$ & $5,506(87.1 \%)$ & $527(14.7 \%)$ & $2,387(78.0 \%)$ & $727(78.7 \%)$ & $254(55.1 \%)$ & $9,616(60.2 \%)$ \\
\hline Prior RT & $12(0.7 \%)$ & $85(1.3 \%)$ & $574(16.0 \%)$ & $355(11.6 \%)$ & $2(0.2 \%)$ & $33(7.2 \%)$ & $1,061(6.6 \%)$ \\
\hline Concurrent RT & $2(0.1 \%)$ & $7(0.1 \%)$ & $175(4.9 \%)$ & $214(7.0 \%)$ & $0(0 \%)$ & $24(5.2 \%)$ & $422(2.6 \%)$ \\
\hline Oral corticosteroid use & $124(7.7 \%)$ & $159(2.5 \%)$ & $502(14 \%)$ & $54(1.8 \%)$ & $33(3.6 \%)$ & $16(3.5 \%)$ & $888(5.6 \%)$ \\
\hline Oral antibiotic use & $687(42.5 \%)$ & $2,474(39.1 \%)$ & $1,648(46.0 \%)$ & $1,649(53.9 \%)$ & $406(43.9 \%)$ & $161(34.9 \%)$ & $7,025(44.0 \%)$ \\
\hline $\begin{array}{l}\text { Selected dermatologic and/or mucosal } \\
\text { conditions }\end{array}$ & $104(6.4 \%)$ & $126(2.0 \%)$ & $115(3.2 \%)$ & $77(2.5 \%)$ & $32(3.46 \%)$ & $23(5.0 \%)$ & $477(3.0 \%)$ \\
\hline $\begin{array}{l}\text { Number of patients who developed FN in the } \\
\text { first cycle of chemotherapy }\end{array}$ & $152(9.4 \%)$ & $268(4.2 \%)$ & $160(4.5 \%)$ & $46(1.5 \%)$ & $27(2.9 \%)$ & $40(8.7 \%)$ & $693(4.3 \%)$ \\
\hline
\end{tabular}

Abbreviations: FN, febrile neutropenia; NHL, non-Hodgkin's lymphoma; RT, radiation therapy.

use of corticosteroids during chemotherapy may also increase risk of FN and should be further evaluated.

Skin and mucosal injuries that breakdown barrier integrity create a point of entry for microorganisms that cause blood stream infections and directly contribute to fever. ${ }^{26}$ In our study cohort, the most common dermatologic and mucosal conditions were gastritis/gastroduodenitis, contact dermatitis, and psoriasis. It is a limitation of this study that we were unable to determine the severity of these conditions using ICD-9 codes. However, it is possible that skin/ mucosal injuries that are more severe or delayed in healing are more likely to be noted and coded by the physician. It has been suggested that neutrophils play an important role in the biological processes of wound healing by serving as first responders in clearing microbes and cellular debris in the wound area. ${ }^{27,28}$ Therefore, in addition to a direct causal role of skin injuries on risk of $\mathrm{FN}$, it is also possible that patients with cancer and impaired neutrophil function are more likely to experience both delayed healing of barrier injuries and FN.

Although we did not find FN risk to have a clear association with oral antibiotic use, we did observe a positive association with intravenous antibiotic use, suggesting that intravenous antibiotics may have a 
Risk Factors for Febrile Neutropenia

Table 3. Adjusted HRs for Oral Corticosteroid Use on Risk for FN

\begin{tabular}{|c|c|c|c|c|c|c|c|}
\hline $\begin{array}{l}\text { Duration } \\
\text { of Use }\end{array}$ & $\begin{array}{l}\text { Patients With FN/Patients } \\
\text { With Corticosteroid } \\
\text { Use (\%) }\end{array}$ & $\mathrm{aHR}^{\mathrm{a}}(95 \% \mathrm{Cl})$ & $P$ Value & Recency of Use & $\begin{array}{l}\text { Patients With FN/ } \\
\text { Patients With } \\
\text { Corticosteroid Use (\%) }\end{array}$ & $\operatorname{aHR}^{\mathrm{a}}(95 \% \mathrm{Cl})$ & $P$ Value \\
\hline No use & $267 / 9,360(2.9 \%)$ & Ref & & No use & $267 / 9,360(2.9 \%)$ & Ref & \\
\hline$<15 d$ & $360 / 5,790(6.2 \%)$ & $1.78(1.50-2.11)$ & $<.01$ & $<15$ d prior to chemotherapy & $403 / 6,082(6.6 \%)$ & $1.88(1.59-2.23)$ & $<.01$ \\
\hline $15-29 d$ & $28 / 436(6.4 \%)$ & $1.84(1.21-2.69)$ & $<.01$ & 15-29 d prior to chemotherapy & $9 / 230(3.9 \%)$ & $1.13(0.54-2.08)$ & .72 \\
\hline $30-44 d$ & $17 / 199(8.5 \%)$ & $2.27(1.33-3.64)$ & $<.01$ & 30-44 d prior to chemotherapy & $5 / 120(4.2 \%)$ & $1.22(0.43-2.66)$ & .66 \\
\hline $45-90 \mathrm{~d}$ & $21 / 186(11.3 \%)$ & $2.86(1.74-4.47)$ & $<.01$ & 45-90 d prior to chemotherapy & $9 / 179(5.0 \%)$ & $1.41(0.67-2.59)$ & .32 \\
\hline
\end{tabular}

Abbreviations: aHR, adjusted hazard ratio; FN, febrile neutropenia; KPSC, Kaiser Permanente Southern California.

${ }^{a}$ Multivariable models adjusted for age (modeled as natural cubic splines), sex, race/ethnicity, length of KPSC membership, public insurance status (Medicare and Medicaid enrollees), and socioeconomic indicators (including census track median annual household income: $\$ 0-\$ 25,000, \$ 25,001-\$ 40,000, \$ 40,001-\$ 60,000, \$ 60,001-\$ 80,000$ and $>\$ 80,000$; and proportion of adults with a high school diploma or a higher degree in the census track: $\leq 50 \%, 51 \%-75 \%,>75 \%$, and prior cancer), stage at diagnosis, congestive heart failure, chronic obstructive pulmonary disease, rheumatoid disease, other autoimmune disease, osteoarthritis, peptic ulcer disease, thyroid disorder, total comorbidity, and recent infection.

more profound impact on the balance of bacterial flora and other immune functions, thus increasing FN risk. However, it is also possible that patients who received intravenous antibiotics were in general sicker and more prone to severe infection. Given that the sample size for this subanalysis was limited (based on 3,199 patients and 236 FN events), the effect of intravenous antibiotic use on FN risk should be confirmed by other studies.

Contrary to our hypotheses, we did not find any association between surgery and RT and risk of FN. It is possible that infection risk associated with these treatments was effectively managed with good wound care or prophylactic antibiotic use. Alternatively, for concurrent RT, it is possible that complications may not have occurred until cycle 2 . It is also possible that those who received surgery and/ or RT were inherently different from those who received chemotherapy alone. For example, those who

\section{Table 4. HRs for Effect of Antibiotic Use on Risk of} FN in the Restricted Analysis (2008-2009)

\begin{tabular}{|c|c|c|c|c|c|}
\hline \multirow{2}{*}{$\begin{array}{l}\text { Antibiotic } \\
\text { Use }\end{array}$} & \multirow{2}{*}{$\begin{array}{l}\text { Number With } \\
\text { FN/Number of } \\
\text { Patients }\end{array}$} & \multicolumn{2}{|c|}{ Bivariate Cox Model } & \multicolumn{2}{|c|}{ PS-Adjusted Modela } \\
\hline & & HR $(95 \% \mathrm{Cl})$ & $P$ Value & HR $(95 \% \mathrm{Cl})$ & $P$ Value \\
\hline No use & $99 / 1,426$ & Ref & & Ref & \\
\hline $\begin{array}{l}\text { Oral use } \\
\text { only }\end{array}$ & $64 / 774$ & $\begin{array}{c}1.16 \\
(0.84-1.58)\end{array}$ & .37 & $\begin{array}{c}1.07 \\
(0.77-1.48)\end{array}$ & .70 \\
\hline $\begin{array}{l}\text { Intravenous } \\
\text { use }\end{array}$ & 73/999 & $\begin{array}{c}1.42 \\
(1.04-1.94)\end{array}$ & .03 & $\begin{array}{c}1.35 \\
(0.97-1.87)\end{array}$ & .08 \\
\hline
\end{tabular}

Abbreviations: FN, febrile neutropenia; HR, hazard ratio; KPSC, Kaiser Permanente Southern California; PS, propensity score.

aMultivariable models adjusted for age (modeled as natural cubic splines), sex, race/ethnicity, length of KPSC membership, public insurance status (Medicare and Medicaid enrollees), and socioeconomic indicators (including census track median annual household income: $\$ 0-\$ 25,000, \$ 25,001-\$ 40,000, \$ 40,001-\$ 60,000$, $\$ 60,001-\$ 80,000$ and $>\$ 80,000$; and proportion of adults with a high school diploma or a higher degree in the census track: $\leq 50 \%, 51 \%-75 \%$, $>75 \%$ ), stage at diagnosis, prior cancer, rheumatoid disease, other autoimmune conditions, osteoarthritis, obesity, diabetes, congestive heart failure, total comorbidity, and recent infection. received surgery may be healthier in general than those who did not. It thus remains possible that the lack of association may be due to confounding from unmeasured factors, such as performance status. Further, because we did not have information on the dose and field of radiation, our null findings do not preclude possible effects of high-dose radiation and/ or direct bone marrow irradiation on FN risk.

Several other potential limitations should be considered. First, our study was limited by the use of an electronic algorithm to capture $\mathrm{FN}$, rather than through chart review. ${ }^{29}$ However, our prior chart review validation demonstrated a positive predictive value of $>90 \%$ of this algorithm (unpublished data). Second, the completeness of ICD-9 coding for the selected dermatologic/mucosal conditions is unknown, and undercoding may be likely due to the temporary and often ill-defined nature of these conditions. Our study may be subject to potential selection bias due to the exclusion of patients who received primary GCSF prophylaxis, which may also explain the relatively lower incidence proportion of FN in our study. Furthermore, we did not examine the dose-response relationship for cumulative doses of corticosteroids or antibiotics. Duration and recentness of use are not entirely independent measures and should not be interpreted as such. We cannot completely exclude the possibility of residual confounding. We also only focused on the first cycle of chemotherapy. However, this restriction was used to avoid potential biases in assessing causal estimates that may occur in subsequent cycles of chemotherapy, such as drop out, switching chemotherapy regimens, or dose reduction due to other related treatment complications. Finally, the study period was not recent. However, this study assessed the etiologic risk factors for FN. The 
Family et al

biology underlying this potential association should not change over time.

\section{Conclusions}

Our study examined several potential novel FN risk factors based on hypothesized underlying pathogenic mechanisms. Our findings suggest conditions that affect the skin/mucosal barrier, corticosteroid use, and intravenous antibiotic use may increase risk of $\mathrm{FN}$, and should be considered in making prophylaxis and patient monitoring decisions. Because patients usually present with multiple FN risk factors, individual patient-level risk factors need to be considered simultaneously to determine each patient's FN risk level. Future research is warranted to develop FN prediction algorithms incorporating established and newly identified patient-level risk factors to help clinicians account for multiple risk factors when making prophylactic decisions.

\section{References}

1. Lyman $\mathrm{GH}$, Lyman $\mathrm{CH}$, Agboola O. Risk models for predicting chemotherapy-induced neutropenia. Oncologist 2005;10:427-437.

2. Chao C, Page JH, Yang SJ, et al. History of chronic comorbidity and risk of chemotherapy-induced febrile neutropenia in cancer patients not receiving G-CSF prophylaxis. Ann Oncol 2014;25:1821-1829.

3. Chia VM, Page JH, Rodriguez R, et al. Chronic comorbid conditions associated with risk of febrile neutropenia in breast cancer patients treated with chemotherapy. Breast Cancer Res Treat 2013;138:621-631.

4. Al-Hadithy $\mathrm{H}$, Isenberg DA, Addison IE, et al. Neutrophil function in systemic lupus erythematosus and other collagen diseases. Ann Rheum Dis 1982;41:33-38.

5. Heinzelmann M, Mercer-Jones MA, Passmore JC. Neutrophils and renal failure. Am J Kidney Dis 1999;34:384-399.

6. Tomer Y, Davies TF. Infection, thyroid disease, and autoimmunity. Endocr Rev 1993;14:107-120.

7. Puchelle E, Zahm JM, Tournier JM, Coraux C. Airway epithelial repair, regeneration, and remodeling after injury in chronic obstructive pulmonary disease. Proc Am Thorac Soc 2006;3:726-733.

8. Miravitlles M, Espinosa C, Fernandez-Laso E, et al. Relationship between bacterial flora in sputum and functional impairment in patients with acute exacerbations of COPD. Study Group of Bacterial Infection in COPD. Chest 1999;116:40-46.

9. Crim C, Calverley PM, Anderson JA, et al. Pneumonia risk in COPD patients receiving inhaled corticosteroids alone or in combination: TORCH study results. Eur Respir J 2009;34:641-647.

10. Jefferies S, Rajan B, Ashley S, et al. Haematological toxicity of craniospinal irradiation. Radiother Oncol 1998;48:23-27.

11. Rapoport BL. Management of the cancer patient with infection and neutropenia. Semin Oncol 2011;38:424-430.

12. Standish LJ, Torkelson C, Hamill FA, et al. Immune defects in breast cancer patients after radiotherapy. J Soc Integr Oncol 2008;6:110-121.

13. Janeway CA Jr, Travers P, Walport M, Sclomchik MJ. Immunobiology: The Immune System in Health and Disease, 5th ed. New York, NY: Garland Science; 1999

14. Turner JR. Intestinal mucosal barrier function in health and disease. Nat Rev Immunol 2009;9:799-809.

15. Gemmell CG. Antibiotics and neutrophil function-potential immunomodulating activities. J Antimicrob Chemother 1993;31(Suppl B):23-33.
16. Rafii F, Sutherland JB, Cerniglia CE. Effects of treatment with antimicrobial agents on the human colonic microflora. Ther Clin Risk Manag 2008;4:1343-1358

17. Butts CL, Sternberg EM. Neuroendocrine factors alter host defense by modulating immune function. Cell Immunol 2008;252:7-15.

18. Lyman GH, Kuderer NM, Crawford J, et al. Predicting individual risk of neutropenic complications in patients receiving cancer chemotherapy. Cancer 2011;117:1917-1927.

19. Hosmer W, Malin J, Wong M. Development and validation of a prediction model for the risk of developing febrile neutropenia in the first cycle of chemotherapy among elderly patients with breast, lung, colorectal, and prostate cancer. Support Care Cancer 2011;19:333-341.

20. Koebnick C, Langer-Gould AM, Gould MK, et al. Sociodemographic characteristics of members of a large, integrated health care system: comparison with US Census Bureau data. Perm J 2012;16:37-41.

21. Gullatte. Clinical Guide to Antineoplastic Therapy: A Chemotherapy Handbook, 2nd ed. Pittsburgh, PA: Oncology Nursing Society; 2007.

22. Baranov AE, Selidovkin GD, Butturini A, Gale RP. Hematopoietic recovery after 10-Gy acute total body radiation. Blood 1994;83:596-599.

23. Jernberg C, Lofmark S, Edlund C, Jansson JK. Long-term impacts of antibiotic exposure on the human intestinal microbiota. Microbiology 2010;156:3216-3223

24. Crawford J, Armitage J, Balducci L, et al. Myeloid growth factors. J Natl Compr Canc Netw 2009;7:64-83.

25. Wright HL, Moots RJ, Bucknall RC, Edwards SW. Neutrophil function in inflammation and inflammatory diseases. Rheumatology (Oxford) 2010;49:1618-1631.

26. van der Velden WJ, Herbers AH, Netea MG, Blijlevens NM. Mucosal barrier injury, fever and infection in neutropenic patients with cancer: introducing the paradigm febrile mucositis. Br J Haematol 2014;167:441452.

27. Broughton G II, Janis JE, Attinger CE. The basic science of wound healing. Plast Reconstr Surg 2006;117(7 Suppl):12s-34s.

28. Campos AC, Groth AK, Branco AB. Assessment and nutritional aspects of wound healing. Curr Opin Clin Nutr Metab Care 2008;11:281-288.

29. Kuderer NM, Wolff AC. Enhancing therapeutic decision making when options abound: toxicities matter. J Clin Oncol 2014;32:1990-1993. 